\title{
Seroprevalencia de la infección por Trypanosoma cruzi en escolares de Villahermosa, Tabasco, México
}

\author{
Enedina Jiménez C. ${ }^{1}$; Guillermina Campos V. ${ }^{1}$; Patricia Ulloa P. ${ }^{2}$; René De la Luz S. ${ }^{1}$
}

\begin{abstract}
Resumen
Objetivo: el propósito de este estudio lue describir la seroprevalencia de la infección por Trypanosoma cruzi en niños menores de 18 años de Villahermosa. Tabasco, y relacionarla con la presencia del vecior. Pacientes y mérodo. en 268 niños, 57,4\% mujeres, se delerminó la presencia de cnticuerpos específicos de la clase lgG para 7 . cruzi en svero por ELISA. Se registró edad, sexo, conocimiento del veclor y antecedentes de contaclo visual con este en su caso o en zonos ruiales. Resultados: la frecuencia total de positividad fue de 3,0\%. Los anticuerpos resultaron positivos en $1,8 \%$ en casos de 4 a 8 años; en $3.9 \%$ de 8 a 12 años; $2.2 \%$ de 12 a 10 años y $4,8 \%$ personas de 10 a 18 años; en $2.0 \%$ de las niñas y $3.5 \%$ de los varones. De los integrontes de la muestra, $13,4 \%$ conocían al vector. $1,5 \%$ lo vio en su casa y $18,7 \%$ en zonas rurales. En:re 51,1 y $58,2 \%$ respordieron no a las lres preguntas y 29,5 a 47,4\% dejaron alguna de ellas sin respuesta. Concírsiones: la seroprevalencia enconlrada fue apenos más elevada que la reporrado por otros autores en medios sirmilares. Lo infección se presentó en rodas las edades predominando en los niños de mavor edad, no huto ielación cor respecto ol sexo. Si bien las respuestos a la encuesia epidemiológiea fueron incompletas, la frecuencia de respuestas afirmotivas fone en evidencia el riesgo de infección en una zona urbono.
\end{abstract}

(Palabras clave: Tryponcsomo cruzi, enfermedad de Chagas, seroprevalencio, vector.)

\section{Seroprevalence of Trypanosoma cruzi infection in school children from Villahermosa city, Tabasco, México}

\begin{abstract}
Objective: to describe the Feecuency of specific antibodies against Tryponosoma cruzi in schoot children aged 18 years or less and to correlale positive serum findings to some epidemiologic information. Subjects and methods: sero of 208 urban school children $57.4 \%$ fernole from villchermasa city, -obasco. Mexico, aged 4 to 18 years old, were investigoted by ELISA for specitic lg class antibodies agoinst $T$. cruzi. Knowledge and visual contact wirh the vector at home or at country side were also recorded. Results: the frequencies of positive serum findings were $3 \%$ as a whole, $2.6 \%$ among female and $3.5 \%$ in male subjects; $1.8 \%$ omong children 4 to 8 years of age; 3.9 among those 8 to 12 years, $2.2 \%$ tor oges 121016 years and $4.8 \%$ fom 10 to 18 years old. Intorming subjects knew the vector in $13.4 \%$ coses, $1.5 \%$ hove sen it at home ond $18.6 \%$ at nearby counlry side, even though almost $60 \%$ of the sample let unanswered all three questions on that matters. Conclusions: this level of seroprevalence is slightly over that reported in other experiences. Evidence of infection was recorded at all ages included in this report and was similar in male and female children. Even though many children didnt answered pertinent questions no less than one third of responders know the vector or have seen it in their surroundings which might be considered an evidence of risk of $T$. cruzi infeclion within urbon populations.
\end{abstract}

(Koy words: Tryponosoma cruzi, Chagos disease.]

La enfermedad de Chagas es una amenaza permanente para la cuarta parte de la población

1. Laboratorio de Investigación en Parasitología. Hospital Infantil de México "Federico Gómez", México, D.F.

2. Hospital Rodolfo Nieto Patrón. Departamento de PatoJogía, Villahermosa, Tabasco, México, D.F. de América Latina'. En México, la tripanosomiasis americana se conoce desde $1940^{2}$, pero sólo en los últimos años ha crecido el interés en el estudio de la enfermedad ${ }^{3}$. Por mucho tiempo se ha especulado sobre la importancia de la misma en nuestro pá́s. Aún existe toda una gama de suposiciones al respecto; desde las que la 
consideran un padecimiento exótico y le niegan cualquier importancia, hasta las que la asumen como un importante problema de salud públi$\mathrm{ca}^{4}$. La realidad es que la enfermedad de Chagas se distribuye en focos dispersos en todas las entidades federativas del país; la gran mayoría de los casos ocurridos se han localizado en regiones de la vertiente del océano Pacífico desde el estado de Sonora hasta Chiapas. Los estados de la República Mexicana en los que se han encontrado más casos humanos de la enfermedad de Chagas diagnosticados serológicamente son los de Chiapas, Yucatán, Zacatecas, Jalisco, Guerrero, Oaxaca y Morelos, lo que señala una transmisión muy activa de la tripanosomiasis en dichas regiones 5 .

Afecta en particular a individuos que habitan en zonas rurales y suburbanas, cuyas viviendas están hechas de adobe con techos de material vegetal y frecuentemente con piso de tierra, donde existen las condiciones adecuadas para que los vectores se reproduzcan ${ }^{6}$.

Como podría comprobarse, la tripanosomiasis americana, como muchas enfermedades parasitarias, está ligada al subdesarrollo econóntico y social. Cabe mencionar que la estructura de la población y los movimientos poblacionales, como la migración del campo a las ciudades e inmigración, constituyen factores de riesgo en la transmisión de la enfermedad en estas regiones?.8.

Otros más son la falta de servicios de salud en las zonas rurales, el no contar con métodos de diagnóstico accesibles para el trabajo de campo, así como las condiciones ecologicas son igualmente factores determinantes en la transmisión de la enfermedad en estas zonas. 10. Más aún, las encuestas estadísticas realizadas en el estado de Tabasco en 1994 por el Sistema Nacional de Salud reportan incremento en los casos nuevos de la enfermedad de Chagas ${ }^{11}$.

Si se estima que el $50 \%$ de la población se encuentra entre 5 a 9 años ${ }^{12}$, podríamos inferir que para conocer la probabilidad de contraer la enfermedad deberemos estudiar la frecuencia de infección en esta población etárea, ya que nos dará una información real porque representa a la mitad de la población total. Por lo que el propósito de este estudio fue determinar la frecuencia en la infección con $T$. cruzi en este grupo de población en Villahermosa, Tabasco.

\section{Pacientes y método}

De acuerdo a la prevalencia reportada ${ }^{13}$ para esta enfermedad, el cálculo de tamaño de la muestra ${ }^{14}$ que deberíamos incluir estudiar fue menor a los 268 nifios estudiados $-154(57,4 \%)$ mujeres-, por lo que consideramos que fue una muestra representaliva. A lodos se les tomó sangre por punción capilar para obtener suero por centrifugación y determinar anticuerpos por triplicado contra $T$. cruzi con la técnica de ELISA. Se consideró como positjva cuando fue mayor al valor de conte antes reportado's. Se formaron grupos de acuerdo a edad y sexo, en todos se realizó la historia epidemiológica, relacionada a que sí conocían o no aj vector, si lo habian visto o no, en su casa, o ел zonas rurales,

Con estos resultados se determinó ta frecuencia de infección de acuerdo a la presencia de anticuerpos específicos y se relacionó con los parametros estudiados, se consideraron diferencias estadísticas cuando el valor de probabilidad fue $\mathrm{p}<0,05$.

\section{Resultados}

El total de muestras positivas fue de ocho ( $3 \%$ ). Se presentaron en todas las edades y predominaron en el grupo de 8 a 12 años. La frecuencia relativa en ellos fue de $4 / 102(3,9 \%)$. No hubo diferencias por sexo (cuatro casos positivos eran niños y cuatro niñas) con frecuencias relativa $2,6 \%$ y $3,5 \%$ respectivamente (tabla 1). En relación a la historia epidemiológica, conocían al vector el $13,4 \%$, lo habían visto en su casa el $1,5 \%$ y lo habían hecho al visitar zonas rurales el $18,7 \%$. Entre el $57-58,3 \%$ respondio no a las tres preguntas y no contestaron a ellas entre el $23,1-47,4 \%$. De los que habían visitado zonas rurales se encontró un caso positivo, esto se muestra en la tabla 2.

La relación entre los sueros positivos para $T$. cruzi y la historia epidemiológica se presenta en la tabla 3 .

\section{Comentario}

En el Estado de Tabasco es notoria la escasez de casos de enfermedad de Chagas si se le compara con lo que se reporta en otros estados de la República Mexicana. Según la encuesta epidemiologica nacional, publicada en 1992, la seroprevalencia ${ }^{13}$ observada en Tabasco de $2,3 \%$ en dilución 1: 8 y por HAI-IFI $1: 32$ fue de $0,1 \%$. Esto parece poco probable, ya que las condiciones climáticas y sociales de este Estado resultan 


\section{Tabla 1}

Frecuencia de la infección por T. cruzi, detectada mediante ELISA en 268 niños

\begin{tabular}{|c|c|c|c|c|c|c|c|c|c|}
\hline \multirow{3}{*}{$\begin{array}{l}\text { Eded } \\
\text { (âfos) }\end{array}$} & \multicolumn{3}{|c|}{ n de examinados } & \multicolumn{4}{|c|}{ Positivos } & \multirow{2}{*}{\multicolumn{2}{|c|}{ Total }} \\
\hline & \multirow[t]{2}{*}{ Hombres } & \multirow[t]{2}{*}{ Mujeres } & \multirow[t]{2}{*}{ Total } & \multicolumn{2}{|c|}{ Hombres } & \multicolumn{2}{|c|}{ Mujeres } & & \\
\hline & & & & $n$ & $\mathscr{F}$ & $n$ & $\%$ & n & $\%$ \\
\hline $4-8$ & 26 & 30 & 56 & $\mathbf{I}$ & 3,8 & 0 & 0.0 & $\mathbf{I}$ & 1.7 \\
\hline $8-12$ & 47 & 55 & 102 & 2 & 4,3 & 2 & 3,6 & 4 & 3.9 \\
\hline $12-16$ & 33 & 56 & 89 & 0 & 0,0 & 2 & 3,6 & 2 & 2,2 \\
\hline $16-18$ & 8 & 13 & 21 & 1 & 12.5 & 0 & 0,0 & $\mathbf{I}$ & 4,8 \\
\hline Total & 314 & 154 & 268 & 4 & 3.5 & 4 & 2,6 & 8 & 3,0 \\
\hline
\end{tabular}

Tabla 2

Conocimiento y contacto visual con el vector reportado por los 268 niños encuestados

\begin{tabular}{lrrrrrrr}
\hline Preguntas & \multicolumn{6}{c}{$\begin{array}{c}\text { Respuestas } \\
\text { Si }\end{array}$} & \multicolumn{2}{c}{ No } & \multicolumn{2}{c}{ No contestó } \\
& n & $\%$ & n & \% & n & $\%$ \\
\hline Conocen al vector & 36 & 13,4 & 153 & 57,1 & 79 & 29,5 \\
Lo vió en st casa & 4 & 1,5 & 137 & 51.1 & 127 & 47,4 \\
Visitan zonas rurales & 50 & 18,7 & 156 & 58.2 & 62 & 23,1 \\
\hline
\end{tabular}

\section{Tabla 3}

Relación entre resultado de ELISA y conocimiento o visualización del vector en los ocho niños positivos

\begin{tabular}{|c|c|c|c|c|}
\hline $\begin{array}{l}\text { Pa- } \\
\text { cjente }\end{array}$ & $\begin{array}{l}\text { DO } \\
\text { Elisa }\end{array}$ & $\begin{array}{c}\text { Conoce al } \\
\text { vector }\end{array}$ & $\begin{array}{c}\text { Lo ha visto } \\
\text { en casa }\end{array}$ & $\begin{array}{l}\text { Visita zonas } \\
\text { rurales }\end{array}$ \\
\hline 1 & $1,772 \pm 0,023$ & No & No & No \\
\hline 2 & $1,999 \pm 0,028$ & No & NR & No \\
\hline 3 & $1,053 \pm 0,031$ & No & No & No \\
\hline 4 & $0,939 \pm 0.027$ & No & No & No \\
\hline 5 & $0,907 \pm 0,033$ & No & No & No \\
\hline 6 & $0,978 \pm 0,04 J$ & No & NR & No \\
\hline 7 & $0,960 \pm 0,018$ & No & $\mathrm{No}$ & Sí \\
\hline 8 & $0,994 \pm 0.040$ & NR & NR & NR \\
\hline
\end{tabular}

Control positivo $1,220 \pm 0,030$

Punto de corte $0,519 \pm 0,144(2 \mathrm{DE})$

DO: densidad óptica. NR: no responde

propicias para suponer que debe haber zonas endémicas de esta enfermedad.

En Tabasco es bien conocida la existencia de numerosos triatominos silvestres y domici- liarios $^{\mathrm{16}}$. La exuberante vegetación, la abundante fauna silvestre y las pobres condiciones económicas prevalentes en gran parte de su población, condicionan que predominen en grupos poblacionales suburbanos y rurales. Gran número de viviendas de esos centros son construidas con carrizo, madera y palmas, consideradas como lípicas de zonas endémicas de enfermedad de Chagas ${ }^{17,18 .}$

Fue por ello que no nos sorprendió en la ciudad de Villahermosa la frecuencia de infección por $T$. cruzi en menores de 19 años $(3,0 \%)$, lo importante es que todos los niños en quienes se efectuó este estudio provienen de una zona urbana, por lo que debe descartarse la idea de que esta sea una enfermedad exclusiva de zonas rurales. Al buscar una explicación encontramos que la mayor frecuencia de positividad se encontró en los niños de 16 a 18 años, y este hecho nos podría sugerir que es en esta edad donde existe mayor facilidad para ir a zonas rurales, al campo, y estar expuestos a la picadura del vector; de tal manera que aunque vivan en zonas urbanas, en algún momento podrían infectarse. Los resultados coinciden con los estudios de prevalencia realizados en países endémi$\cos ^{19}$, donde se reporta mayor prevalencia de infección en grupos de esta edad, en que se habla del $17,4 \%$.

Este planteamiento concuerda con la historia epidemiologica donde $13.4 \%$ conocia al vector, puesto que el $18,6 \%$ salía a zonas rurales. No obstante, la segunda pregunta refuerza la idea de considerar que el vector está en una zona urbana, ya que el 1,5\% lo vio en su casa. Cabe mencionar que fue difícil analizar estos resultados porque más del $50 \%$ respondió no o bien no contestó a estas preguntas, lo cual nos parece 
muy explicable tratándose de niños; si lo hubiesen hecho, probablemente los casos positivos confirmarían que sí conocían al vector, que lo habían visto en su casa y con ello la infección proviene de un triatona infectado localizado en una zona urbana que pasó desapercibida. Nos permitimos hacer ese planteamiento porque a nuestro juicio el riesgo de infectarse con $T$. cru$z i$ no ha sido estudiado cabalmente. Hasta 1986 , sólo aparecían en las publicaciones médicas dos casos confirmados de cardiopatía chagásica procedentes de Tabasco ${ }^{20,21}$. Posteriormente en una encuesta seropidemiológica con muestras aleatorias en una comunidad del municipio de Macuspana, se obtuvo $13 \%$ de seroinfección por T. cruzi (HAJ-IFI). Demostrándose además la existencia de triatomas infectados con $T$. cru$z i^{22}$. En el Hospital del Niño Rodolfo Nieto Patrón, en trabajo de Tesis, se demostró $50 \%$ de serología positiva para enfermedad de Chagas en casos de cardiopatía dilatada ${ }^{23}$, y se ha demostrado parasitoscópicamente en autopsias cuatro casos ${ }^{24}$.

El que la infección no se manifieste como consecuencia de la poca sintomatología, favorece que en muchos casos pase desapercibida, lo cual se explica por dos razones: la primera es la importancia de los mecanismos de defensa del huésped que pueden regular la virulencia del parásito ${ }^{25}$, y la segunda es que se infecten con cepas de diferentes patogenicidad. Finalmente, consideramos que nuestros resultados son importantes porque el valor de prevalencia de infección fue mayor al reportado anteriormente. Este incremento nos pone en alerta, porque el vector se encuentra en la zona urbana y probablemente aquel se haga mayor si no hay control del vector.

\section{Referencias}

I. OMS: Control de la enfermedad de Chagas. Informe de un Comité de Expertos de la OMS. Serie Inforınes Técnicos $N^{0} 811$, Ginebra. 1991: 101 .

2. Mazzonti L: Dos casos de la enfermedad de Chagas en el Eslado de Oaxaca. Gac Med Mex 1940; 70: 417420.

3. Reyes PA: Chagas disease in North America. Am Heart J 1993: 126: 1496.

4. Velazeo $C O$ : La enfermedad de Chagas en México. Infectología 1992; 12: 783-791.

5. Tay J, Schenone H, Sánchez J.T, Robert L: Estado actual de los conocimientos sobre la enfermedad de
Chagas en la República Mexicana. Bol Chil Parasitol $1992 ; 47 ; 43-53$.

6. Mott KE, Muniz TM, Lehman JS, et al: Household distribution of seroreactivity to Trypanesomea cruzi in in rural community Northeast Brasil. Am J Trop Med Hyg 1978: $27: 1116.1122$.

7. OPS: La Historia nalural, la epidemiología y el control de la enferınedad de Chagas. 1984; serie $\mathrm{N}^{\circ} 92$.

8. Godal $T:$ Special programme for research and training in tropical disease, World Health Organization. Geneva, Switzerland. The roles of the World Health Organization in Tropical disease research. Ann $N$ Y Acad Sci 1989: 569: 318-324.

9. Reyes PA, Monteon V. Pérez R. et al: Estudio de la en. fermedad de Chagas en una comunidad del Estado de Puebla. Mem. XI Congreso Nacional de Parasitología. Querétaro. Qro. 1994

10. Uttos HF; Geografía de la enfennedad de Chagas en el Estado de Morelos. Tesis de Licenciatura. Facultad de Filosofia y Letras (UNAM), 1989.

11. Sistena Nacionat de Stibd (Mexico): Casos nuevos de enfermedades. Boletín de Información Estadistica. 1994; 2: 35-39.

12. INEGI, Tabasco: Resultados definitivos: tabuladores básicos conteo de población y vivienda, 1995; 78; 101-103.

13. Castrejon-Velasco O. Vatdespino JL, Tapia-Conyer $R$, et al: Seroepidemiología de la enfermedad de Chagas en México. Salud Pública en México 1992: 34: 186 196.

14. Demenech JM: Bipestadística, métodos estadísticos para investigadores. Editorial Herder, Barcelona 1982: $70-7 \mathrm{i}$.

15. Jimênez-Cardoso $E$, De la Luz $R$, Campos $G$. Prevalencia de infección por $T$. cruzi en niños asinto. máticos de 4 a 12 afos de edad mediante la Técnica de ELISA. Rev Enf Inf en Pediattía 1995; 9: 2-8.

16. Tay J. Salazar-Schenino PM. Bucio $M$, Zárate $R$. Zirate L: La enfermedad de Chagas en la República Mexicana. Sal Pub Mex 1980: 22: 409-450

17. Edgcomb $J H$. Johnson CM: American Trypanosomiasis. In: Diseases caused by prolozoa Ed: BINFOR'S. Chil Atlas extraordinary diseases. (Arm For Inst Path) Washington D.C. 1976: 244-251.

18. Toro $G$, Román $G$. In: Tripanosomiasis Americana (enfermedad de Chagas) en Protozoarios y Helininlos. Cap IV. Neuropatología Tropical. I' cdición. Bogotá, Colombia Ltda., 1983: 118 - 119.

19. Baruffo $G$. Alcántara $F$ : Prevalencia serologica de doença de Chagas na zona sul do Rio Grande do Sul (Brasil). Rev Inst Med Trop Soc Paulo. 1977: 19: 117. 123.

20. Velasco-Casirejon O, Guzmá-Bracho C: Importancia de la enfermedad de Chagas en México. Rev Lal Amer Microbiologia 1986; $28: 275.286$.

21. Marcuchamer-Milier J. Reyes-Lopez P: Enfermedad de Chagas en Mexico. Reporte de 5 casos comprobados. Arch lnst Card Mex 1978; 4: 952-963.

22. Sánchez B, Velasco-Castrejón O: Estudio Seroepidemiologico de la enfermedad de Chagas en Benito Juárez, Macuspana. Tabasco. Rey Med UJAT 1989; 3: 4.6. 
23. Herrera-Camino WR. UHoa PP: Cardiopalía dilatada y enfermedad de Chagas en el Hospital del Niño. Tesis de Postgrado. Hospital Rodolfo Nieto Patrón, Villahermosa, Tabasco 1995.

24. Ulloa-Patimo P, Bulnes-Mendizábal D: Miocarditis Chagásica. Hallazgos en cuatro casos de autopsjas pediátricas. Memorias [II Reunión Nal. de Chagas. Inst Nac de Cardiología, Dr. Ignacio Chávez 1992.

25. Tall BF. Joiner KA: Developmentally regulated virulence factors of Trypanosuma cruzi and there relationship to evasion of host defences. J Eukaryot Microbiol 1993; 40: 207-213.

Esta publicación está disponible en copias de microfilms de $16 \mathrm{y}$ $35 \mathrm{~mm}$ y microfichas de $105 \mathrm{~mm}$, las que pueden solicitarse a:

University Microfilms International 300 North Zeeb Road Ann Arbor, Michigan 48106, USA.

This journal is also available in $16 \mathrm{~mm}$ microfilm, $35 \mathrm{~mm}$ microfilm and $105 \mathrm{~mm}$ microfilm copies through:

University Microfilms International, 300 North Zeeb Road, Ann Arbor, Michigan 48106. USA. 